\title{
Existence and Uniform Decay of Solutions of a Parabolic-Hyperbolic Equation with Nonlinear Boundary Damping and Boundary Source Term ${ }^{1}$
}

\author{
M.M. Cavalcanti, V.N. Domingos Cavalcanti, \\ J.S. PRATES Filho and J.A. Soriano
}

Existence and uniform decay of solutions of a mixed problem based on the degenerate equation

$$
K_{1}(x, t) y_{t t}+K_{2}(x, t) y_{t}-\Delta_{x} y=0
$$

are studied. Under the assumptions that we have a nonlinear boundary damping $\left(1+\alpha(t)\left|y_{t}\right|^{\rho}\right) y_{t}$ and a boundary source term of type $\alpha(t)|y|^{\gamma} y$, we establish the global existence theorem provided $\rho \geq \gamma$ and we obtain the uniform decay of strong and weak solutions considering $\rho=\gamma$ and the coefficient $\alpha(t)$ producing a damping effect.

\section{Introduction.}

Throughout, $\Omega$ will be a bounded domain of $R^{n}$ with $C^{2}$ boundary $\Gamma, \Gamma=$ $\Gamma_{0} \cup \Gamma_{1}$, with both $\Gamma_{0}$ and $\Gamma_{1}$ having positive measure. With this geometry, we shall consider here the following problem

$(1.1) \begin{cases}K_{1}(x, t) y_{t t}+K_{2}(x, t) y_{t}-\Delta_{x} y=0 & \text { in } Q=\Omega \times(0, \infty) \\ y=0 & \text { on } \Sigma_{1}=\Gamma_{1} \times(0, \infty) \\ \frac{\partial y}{\partial \nu}+y_{t}+\alpha(t)\left(\left|y_{t}\right|^{\rho} y_{t}-|y|^{\gamma} y\right)=0 & \text { on } \Sigma_{0}=\Gamma_{0} \times(0, \infty) \\ y(x, 0)=y^{0}(x) \text { and } y_{t}(x, 0)=y^{1}(x) & \text { for } x \in \Omega .\end{cases}$

\footnotetext{
${ }^{1}$ Research partially supported by a grant of CNPq-Brazil.
} 
where

$$
0<\gamma, \rho \leq \frac{1}{n-2} \quad \text { if } \quad n \geq 3 \quad \text { or } \quad \gamma, \rho>0 \quad \text { if } \quad n=1,2
$$

and $\nu$ denotes the unit outward normal vector to the boundary.

The main goal of this paper is to study the existence and uniform decay of solutions to (1.1), assuming that $K_{1}(x, t)$ can vanish on $Q$. When $\alpha(t)$ acts as a damping mechanism and $\rho \geq \gamma$, we prove existence of strong and weak solutions to (1.1), when $\rho=\gamma$ the uniform decay of the energy

$$
e(t)=\frac{1}{2} \int_{\Omega} K_{1}(x, t)\left|y_{t}(x, t)\right|^{2} d x+\int_{\Omega}|\nabla y(x, t)|^{2} d x
$$

is obtained.

This kind of problem is specially related to the study of transonic gas dynamics, see e.g., Lar'kin [8]. Nondegenerate evolution equations with nonlinear feedbaks acting on the boundary have received considerable attention and in this direction we refer the works of Lagnese and Leugering [7], Lasiecka and Tataru [10], Zuazua [13] and references therein. Concerning nonlinear damping and source terms acting on the domain we refer the work of Georgiev and Todorova [5]. The existence and boundary stabilization of solutions to degenerate evolution equations were early considered in Literature (see Cavalcanti et al. [1, 2]). The present problem deals with degenerate evolution equations and nonlinear boundary feedback combined with a nonlinear boundary source term. This was not previously considered in Literature and brings up new difficulties.

The existence of solutions is obtained from the Faedo-Galerkin method (see Lions [11]) and the uniform stabilization is proved by using the perturbed energy method (see Zuazua [13]).

Our paper is organized as follows. In section 2 we give some notations and state our main result. In section 3 we obtain existence of strong solutions to problem (1.1) and in section 4 we obtain the uniform decay of the energy.

\section{Assumptions and Main Result.}

We define

$$
\begin{gathered}
V=\left\{u \in H^{1}(\Omega) ; u=0 \text { on } \Gamma_{1}\right\} \\
(u, v)=\int_{\Omega} u(x) v(x) d x,(u, v)_{\Gamma_{0}}=\int_{\Gamma_{0}} u(x) v(x) d \Gamma
\end{gathered}
$$




$$
\begin{gathered}
|u|^{2}=\int_{\Omega}|u(x)|^{2} d x ;|u|_{\Gamma_{0}}^{2}=\int_{\Gamma_{0}}|u(x)|^{2} d \Gamma \\
\|u\|_{p, \Gamma_{0}}=\left(\int_{\Gamma_{0}}|u(x)|^{p} d \Gamma\right)^{1 / p},\|u\|_{\infty}=\operatorname{ess} \sup _{t \geq 0}\|u(t)\|_{L^{\infty}(\Omega)} .
\end{gathered}
$$

The variational formulation associated with problem (1.1) is given by

$$
\begin{aligned}
& \left(K_{1}(t) y_{t t}(t), w\right)+\left(K_{2}(t) y_{t}(t), w\right)+(\nabla y(t), \nabla w)+\left(y_{t}(t), w\right)_{\Gamma_{0}} \\
& \quad+\alpha(t)\left(\left|y_{t}(t)\right|^{\rho} y_{t}(t), w\right)_{\Gamma_{0}} \\
& =\alpha(t)\left(|y(t)|^{\gamma} y(t), w\right)_{\Gamma_{0}}, \forall w \in V .
\end{aligned}
$$

In order to obtain the existence of solutions we consider $w=y_{t}(t)$. Concerning strong solutions, an additional estimate is needed, that is, the one obtained by derivating the variational formulation (2.2) with respect to $t$. In view of the surface integrals, it is not suitable the use of a special basis, for instance, those formed by eigenfunctions. But the presence of the term $\left|y_{t t}(0)\right|$ leads us to technical problems. To solve this question we assume that

$$
K_{1}(x, 0) \geq d>0 \text { a.e in } Q
$$

and we make the following compatibility hypotheses upon the initial data.

\section{(A.1) Assumptions on the Initial Data.}

Let us consider

$$
y^{0}, y^{1} \in V \cap H^{2}(\Omega)
$$

verifying the compatibility condition

$$
\frac{\partial y^{0}}{\partial \nu}+y^{1}+\alpha(0)\left(\left|y^{1}\right|^{\rho} y^{1}-\left|y^{0}\right|^{\gamma} y^{0}\right)=0 \text { on } \Gamma_{0} .
$$

We observe that even in the linear case, it is not clear that hypothesis (H.1) and (H.2) imply the boundness of $\left|y_{t t}(0)\right|$. In fact, in order to notice it let us transform problem (1.1) into an equivalent one with null initial data. More precisely, defining

$$
\phi(x, t)=y^{0}(x)+t y^{1}(x) ; \quad(x, t) \in \Omega \times(0, \infty)
$$

and

$$
v(x, t)=y(x, t)-\phi(x, t)
$$


454 M.M. Cavalcanti, V.N.D. Cavalcanti, J.S.P. Filho \& J.A. Soriano

we obtain the equivalent problem for $v$

$$
\begin{cases}K_{1} v_{t t}+K_{2} v_{t}-\Delta v=F & \text { in } Q \\ v=0 & \text { on } \Sigma_{1} \\ \frac{\partial v}{\partial \nu}+v_{t}+\alpha(t)\left(\left|v_{t}+\phi_{t}\right|^{\rho}\left(v_{t}+\phi_{t}\right)-|v+\phi|^{\gamma}(v+\phi)\right)=G & \text { on } \Sigma_{0} \\ v(0)=v_{t}(0)=0 & \end{cases}
$$

where

$$
F=-K_{2} \phi_{t}+\Delta \phi \quad \text { and } \quad G=-\frac{\partial \phi}{\partial \nu}-\phi_{t}
$$

The new variational formulation associated with (2.5) is given by

$$
\begin{aligned}
& \left(K_{1}(t) v_{t t}(t), w\right)+\left(K_{2}(t) v_{t}(t), w\right)+(\nabla v(t), \nabla w)+\left(v_{t}(t), w\right)_{\Gamma_{0}} \\
& \quad+\alpha(t)\left(\left|v_{t}(t)+\phi_{t}(t)\right|^{\rho}\left(v_{t}(t)+\phi_{t}(t)\right), w\right)_{\Gamma_{0}} \\
& =\alpha(t)\left(|v(t)+\phi(t)|^{\gamma}(v(t)+\phi(t)), w\right)_{\Gamma_{0}} \\
& \quad+(F(t), w)+(G(t), w)_{\Gamma_{0}} .
\end{aligned}
$$

Considering $w=v_{t t}(0)$ in equation (2.7) from (H.1), (H.2), (2.6) and taking into account that $v(0)=v^{\prime}(0)=0$ we conclude that there exists $C>0$ such that

$$
\left|v_{t t}(0)\right|^{2} \leq C
$$

Next, we are going to consider

\section{(A.2) Assumptions on the Coefficients.}

Let us assume that

$$
\begin{gathered}
K_{1}, K_{2} \in W^{1, \infty}\left(0, \infty ; L^{\infty}(\Omega)\right), \\
K_{2}-\frac{1}{2}\left|K_{1, t}\right| \geq \delta>0 \text { a.e. in } Q .
\end{gathered}
$$

The hypothesis (H.4) was widely used in degenerate problems. We refer the reader to the works of the authors Lar'kin et al. [8] and Cavalcanti et al. [2]. 


\section{(A.3) Assumptions on the Coefficient $\alpha$.}

Let us consider

$$
\alpha \in W^{1, \infty}(0, \infty) \cap L^{1}(0, \infty), \quad \alpha \geq 0,
$$

verifying

$$
-m_{0} \alpha(t) \leq \alpha_{t}(t) \leq-m_{1} \alpha(t) \text { for all } t \geq 0
$$

for some $m_{0}, m_{1}>0$.

Now we are in position to state our main result.

Theorem 2.1. Under the assumptions (A.1), (A.2), (A.3) and assuming that $\gamma, \rho$ satisfy the hypothesis (1.2) with $\rho \geq \gamma$, problem (1.1) has a unique strong solution $y: \Omega \rightarrow \mathbf{R}$ verifying

$$
\begin{gathered}
y \in L^{\infty}(0, \infty ; V) \quad \text { and } y^{\prime} \in L^{\infty}(0, \infty ; V), \\
\sqrt{K_{1}} y^{\prime \prime} \in L^{\infty}\left(0, \infty ; L^{2}(\Omega)\right) \quad \text { and } y^{\prime \prime} \in L^{2}\left(0, \infty ; L^{2}(\Omega)\right), \\
K_{1} y^{\prime \prime}+K_{2} y^{\prime}-\Delta y=0 \quad \text { in } \quad Q \\
y=0 \quad \text { on } \Sigma_{1}, \\
\frac{\partial y}{\partial \nu}+y^{\prime}+\alpha(t)\left(\left|y^{\prime}\right|^{\rho} y^{\prime}-|y|^{\gamma} y\right)=0 \quad \text { on } \Sigma_{0}, \\
y(0)=y^{0} \quad \text { and } \quad y^{\prime}(0)=y^{1} \text { on } \Omega .
\end{gathered}
$$

Moreover, if $\rho=\gamma$ and $m_{1}$ is large enough, there exists a positive constant $\varepsilon_{0}$ such that

$$
E(t) \leq 3 \exp \left(-\frac{\varepsilon}{2} t\right), \quad \forall t \geq 0 \quad \text { and } \quad \forall \varepsilon \in\left(0, \varepsilon_{0}\right] .
$$

Theorem 2.2. Assume that assumptions (H.1), (A.2) and (H.5) hold; consider $\alpha(0)=0$ and that (H.6) holds for all $t \in\left(t_{0},+\infty\right)$. Then, given $\left\{y^{0}, y^{1}\right\} \in V \times L^{2}(\Omega)$, problem (1.1) possesses at least a solution in the class

$$
y \in C^{0}([0, \infty) ; V) \cap C^{1}\left([0, \infty) ; L^{2}(\Omega)\right) .
$$

In addition, we obtain the same uniform decay rates given in (2.11) for the weak solution and for all $t \geq t_{0}$. 


\section{Existence and Uniqueness of Solutions.}

In this section we are going to obtain existence and uniqueness of strong and weak solutions to problem (1.1) using the Faedo-Galerkin method. For this end we represent by $\left\{\omega_{j}\right\}_{j \in \mathbf{N}}$ a basis in $V \cap H^{2}(\Omega)$ which is orthonormal in $L^{2}(\Omega)$, by $V_{m}$ the subspace of $V$ generate by the first $m$ vectors $\left\{w_{1}, \cdots, w_{n}\right\}$ and we define for each $\varepsilon>0$

$$
K_{1, \varepsilon}=K_{1}+\varepsilon \quad \text { and } \quad v_{\varepsilon m}(t)=\sum_{j=1}^{m} g_{\varepsilon j m}(t) \omega_{j},
$$

where $v_{\varepsilon m}(t)$ is the solution of the following Cauchy problem

$$
\begin{aligned}
& \left(K_{1, \varepsilon}(t) v_{\varepsilon m}^{\prime \prime}(t), w\right)+\left(K_{2}(t) v_{\varepsilon m}^{\prime}(t), w\right)+\left(\nabla v_{\varepsilon m}(t), \nabla w\right)+\left(v_{\varepsilon m}^{\prime}(t), w\right)_{\Gamma_{0}} \\
& \quad+\alpha(t)\left(\left|v_{\varepsilon m}^{\prime}(t)+\phi^{\prime}(t)\right|^{\rho}\left(v_{\varepsilon m}^{\prime}(t)+\phi^{\prime}(t)\right), w\right)_{\Gamma_{0}} \\
& =\alpha(t)\left(\left|v_{\varepsilon m}(t)+\phi(t)\right|^{\gamma}\left(v_{\varepsilon m}(t)+\phi(t)\right), w\right)_{\Gamma_{0}} \\
& \quad+(F(t), w)+(G(t), w)_{\Gamma_{0}}, \quad \forall w \in V_{m}
\end{aligned}
$$

$$
v_{\varepsilon m}(0)=v_{\varepsilon m}^{\prime}(0)=0
$$

The above approximate system is a normal one of differential equations which has solution in [0, $T_{\varepsilon m}$ [. The extension of these solutions to the whole interval $[0, T]$ is a consequence of the first estimate which we are going to prove below.

\section{A Priori Estimates.}

\section{The First Estimate.}

Replacing $w$ by $v_{\varepsilon m}^{\prime}(t)$ in (3.2) we obtain

$$
\begin{aligned}
\frac{d}{d t} & \left\{\frac{1}{2}\left|\sqrt{K_{1, \varepsilon}(t)} v_{\varepsilon m}^{\prime}(t)\right|^{2}+\frac{1}{2}\left|\nabla v_{\varepsilon m}(t)\right|^{2}+\frac{\alpha(t)}{\gamma+2}\left\|v_{\varepsilon m}(t)+\phi(t)\right\|_{\gamma+2, \Gamma_{0}}^{\gamma+2}\right\} \\
& +\left(K_{2}-\frac{1}{2} K_{1}^{\prime}(t), v_{\varepsilon m}^{\prime 2}(t)\right)+\alpha(t)\left\|v_{\varepsilon m}^{\prime}(t)+\phi^{\prime}(t)\right\|_{\rho+2, \Gamma_{0}}^{\rho+2}+\left|v_{\varepsilon m}^{\prime}(t)\right|_{\Gamma_{0}}^{2} \\
= & 2 \alpha(t)\left(\left|v_{\varepsilon m}(t)+\phi(t)\right|^{\gamma}\left(v_{\varepsilon m}(t)+\phi(t)\right),\left(v_{\varepsilon m}^{\prime}(t)+\phi^{\prime}(t)\right)\right)_{\Gamma_{0}}
\end{aligned}
$$




$$
\begin{aligned}
& +\frac{1}{\gamma+2} \alpha^{\prime}(t)\left\|v_{\varepsilon m}(t)+\phi(t)\right\|_{\gamma+2, \Gamma_{0}}^{\gamma+2} \\
& +\alpha(t)\left(\left|v_{\varepsilon m}^{\prime}(t)+\phi^{\prime}(t)\right|^{\rho}\left(v_{\varepsilon m}^{\prime}(t)+\phi^{\prime}(t)\right), \phi^{\prime}(t)\right)_{\Gamma_{0}} \\
& -\alpha(t)\left(\left|v_{\varepsilon m}(t)+\phi(t)\right|^{\rho}\left(v_{\varepsilon m}(t)+\phi(t)\right), \phi^{\prime}(t)\right)_{\Gamma_{0}} \\
& +\left(F(t), v_{\varepsilon m}^{\prime}(t)\right)+\left(G(t), v_{\varepsilon m}^{\prime}(t)\right)_{\Gamma_{0}} .
\end{aligned}
$$

Making use of Young's inequality $a b \leq C(\eta) a^{p}+\eta b^{q}$, where $\frac{1}{p}+\frac{1}{q}=1$ and $\eta$ is an arbitrary positive number, considering the assumptions (H.4) and (H.6), from (3.4) we infer

$$
\begin{aligned}
& \frac{d}{d t}\left\{\frac{1}{2}\left|\sqrt{K_{1, \varepsilon}(t)} v_{\varepsilon m}^{\prime}(t)\right|^{2}+\frac{1}{2}\left|\nabla v_{\varepsilon m}(t)\right|^{2}+\frac{\alpha(t)}{\gamma+2}\left\|v_{\varepsilon m}(t)+\phi(t)\right\|_{\gamma+2, \Gamma_{0}}^{\gamma+2}\right\} \\
& +(\delta-\eta)\left|v_{\varepsilon m}^{\prime}(t)\right|^{2}+(1-\eta)\left|v_{\varepsilon m}^{\prime}(t)\right|_{\Gamma_{0}}^{2}+(1-\eta) \alpha(t)\left\|v_{\varepsilon m}^{\prime}(t)+\phi^{\prime}(t)\right\|_{\rho+2, \Gamma_{0}}^{\rho+2} \\
& \leq \frac{1}{4 \eta}|F(t)|^{2}+\frac{1}{4 \eta}|G(t)|_{\Gamma_{0}}^{2}+\eta \alpha(t)\left\|v_{\varepsilon m}^{\prime}(t)+\phi^{\prime}(t)\right\|_{\gamma+2, \Gamma_{0}}^{\gamma+2} \\
& +C_{1}(\eta) \alpha(t)\left\|v_{\varepsilon m}(t)+\phi(t)\right\|_{\gamma+2, \Gamma_{0}}^{\gamma+2}+C_{2}(\eta,\|\alpha\|)\left\|y^{1}\right\|_{\rho+2, \Gamma_{0}}^{\rho+2} \\
& +\alpha(t)\left\|v_{\varepsilon m}(t)+\phi(t)\right\|_{\gamma+2, \Gamma_{0}}^{\gamma+2}+C_{3}(\|\alpha\|)\left\|y^{1}\right\|_{\gamma+2, \Gamma_{0}}^{\gamma+2} .
\end{aligned}
$$

Estimate for $I:=\eta \alpha(t)\left\|v_{\varepsilon m}^{\prime}(t)+\phi^{\prime}(t)\right\|_{\gamma+2, \Gamma_{0}}^{\gamma+2}$. write

$$
|I| \leq \eta C_{4} \alpha(t)+\eta \alpha(t) C_{4}\left\|v_{\varepsilon m}^{\prime}(t)+\phi^{\prime}(t)\right\|_{\rho+2, \Gamma_{0}}^{\rho+2},
$$

where $C_{4}$ is a positive constant independent of $\varepsilon$ and $m$.

Combining (3.5) and (3.6), integrating the obtained result over $[0, t]$ taking (3.3) into account, employing Gronwall's lemma and choosing $\eta>0$ sufficiently small we obtain the first estimate

$$
\begin{aligned}
& \left|\sqrt{K_{1, \varepsilon}(t)} v_{\varepsilon m}^{\prime}(t)\right|^{2}+\left|\nabla v_{\varepsilon m}(t)\right|^{2}+\alpha(t)\left\|v_{\varepsilon m}(t)+\phi(t)\right\|_{\gamma+2, \Gamma_{0}}^{\gamma+2} \\
& \quad+\int_{0}^{t}\left|v_{\varepsilon m}^{\prime}(s)\right|^{2} d s+\int_{0}^{t}\left|v_{\varepsilon m}^{\prime}(s)\right|_{\Gamma_{0}}^{2} d s \\
& \quad+\int_{0}^{t} \alpha(s)\left\|v_{\varepsilon m}^{\prime}(s)+\phi^{\prime}(s)\right\|_{\rho+2, \Gamma_{0}}^{\rho+2} d s \leq L_{1},
\end{aligned}
$$


where $L_{1}>0$ is independent of $\varepsilon$ and $m$.

\section{The Second Estimate.}

Differentiating (3.2) and substituing $w$ by $v_{\varepsilon m}^{\prime \prime}(t)$, we have

$$
\begin{aligned}
\frac{d}{d t} & \left\{\frac{1}{2}\left|\sqrt{K_{1, \varepsilon}(t)} v_{\varepsilon m}^{\prime \prime}(t)\right|^{2}+\frac{1}{2}\left|\nabla v_{\varepsilon m}^{\prime}(t)\right|^{2}\right\}+\left(K_{2}(t)+\frac{1}{2} K_{1}^{\prime}, v_{\varepsilon m}^{\prime \prime 2}(t)\right) \\
& +\left(K_{2}(t) v_{\varepsilon m}^{\prime}(t), v_{\varepsilon m}^{\prime \prime}(t)\right)+\left|v_{\varepsilon m}^{\prime \prime}(t)\right|_{\Gamma_{0}}^{2} \\
& +\alpha^{\prime}(t)\left(\left|v_{\varepsilon m}^{\prime}(t)+\phi^{\prime}(t)\right|^{\rho}\left(v_{\varepsilon m}^{\prime}(t)+\phi^{\prime}(t)\right), v_{\varepsilon m}^{\prime \prime}(t)\right)_{\Gamma_{0}} \\
& +(\rho+1) \alpha(t)\left(\left|v_{\varepsilon m}^{\prime}(t)+\phi^{\prime}(t)\right|^{\rho}, v_{\varepsilon m}^{\prime \prime 2}(t)\right)_{\Gamma_{0}} \\
= & \alpha^{\prime}(t)\left(\left|v_{\varepsilon m}(t)+\phi(t)\right|^{\gamma}\left(v_{\varepsilon m}(t)+\phi(t)\right), v_{\varepsilon m}^{\prime \prime}(t)\right)_{\Gamma_{0}} \\
& +(\gamma+1) \alpha(t)\left(\left|v_{\varepsilon m}(t)+\phi(t)\right|^{\gamma}\left(v_{\varepsilon m}^{\prime}(t)+\phi^{\prime}(t)\right), v_{\varepsilon m}^{\prime \prime}(t)\right)_{\Gamma_{0}} \\
& +\left(F^{\prime}(t), v_{\varepsilon m}^{\prime \prime}(t)\right)+\left(G^{\prime}(t), v_{\varepsilon m}^{\prime \prime}(t)\right)_{\Gamma_{0}} .
\end{aligned}
$$

Estimate for $I_{1}:=\alpha^{\prime}(t)\left(\left|v_{\varepsilon m}^{\prime}(t)+\phi^{\prime}(t)\right|^{\rho}\left(v_{\varepsilon m}^{\prime}(t)+\phi^{\prime}(t)\right), v_{\varepsilon m}^{\prime \prime}(t)\right)_{\Gamma_{0}}$.

From assumption (H.6) and using the inequality $a b \leq \frac{1}{4 \eta} a^{2}+\eta b^{2}, \eta>0$, we conclude

$$
\begin{aligned}
\left|I_{1}\right| \leq & \frac{m_{0} \alpha(t)}{4 \eta}\left\|v_{\varepsilon m}^{\prime}(t)+\phi^{\prime}(t)\right\|_{\rho+2, \Gamma_{0}}^{\rho+2} \\
& +m_{0} \eta \alpha(t)\left(\left|v_{\varepsilon m}^{\prime}(t)+\phi(t)\right|^{\rho}, v_{\varepsilon m}^{\prime \prime 2}(t)\right) .
\end{aligned}
$$

Estimate for $I_{2}:=\alpha^{\prime}(t)\left(\left|v_{\varepsilon m}(t)+\phi(t)\right|^{\gamma}\left(v_{\varepsilon m}(t)+\phi(t)\right), v_{\varepsilon m}^{\prime \prime}(t)\right)_{\Gamma_{0}}$.

Taking into account that $\frac{\gamma}{2 \gamma+2}+\frac{1}{2 \gamma+2}+\frac{1}{2}=1$, using the generalized Hölder inequality, the continuity of the trace operator $\gamma_{0}: H^{1}(\Omega) \rightarrow L^{q}(\Gamma)$, for $1 \leq q \leq \frac{2 n-2}{n-2}$, and the first estimate, it follows that

$$
\begin{aligned}
\left|I_{2}\right| & \leq C_{5}\left\|v_{\varepsilon m}(t)+\phi(t)\right\|_{\gamma+2, \Gamma_{0}}^{\gamma} \| v_{\varepsilon m}(t)+\left.\phi(t)\right|_{2 \gamma+2, \Gamma_{0}}\left|v_{\varepsilon m}^{\prime \prime}(t)\right|_{\Gamma_{0}} \\
& \leq C_{6}(T, \eta)\left|\nabla v_{\varepsilon m}(t)\right|^{2}+\eta\left|v_{\varepsilon m}^{\prime \prime}(t)\right|^{2} \\
& \leq C_{7}(T, \eta)+\eta\left|v_{\varepsilon m}^{\prime \prime}(t)\right|^{2} .
\end{aligned}
$$

Estimate for $I_{3}=(\gamma+1) \alpha(t)\left(\left|v_{\varepsilon m}(t)+\phi(t)\right|^{\gamma}\left(v_{\varepsilon m}^{\prime}(t)+\phi^{\prime}(t)\right), v_{\varepsilon m}^{\prime \prime}(t)\right)_{\Gamma_{0}}$.

Considering the same arguments used in (3.10) we obtain

$$
\left|I_{3}\right| \leq C_{8}(T, \eta)\left|\nabla v_{\varepsilon m}^{\prime}(t)\right|^{2}+\eta\left|v_{\varepsilon m}^{\prime \prime}(t)\right|^{2} .
$$


Making use of assumption (H.4), combining equations (3.8)-(3.10), integrating over $[0, t]$ the obtained result taking equation (2.7) into account, employing Gronwall's lemma and choosing $\eta$ small enough we obtain the second estimate

$$
\begin{aligned}
& \left|\sqrt{K_{1, \varepsilon}(t)} v_{\varepsilon m}^{\prime \prime}(t)\right|^{2}+\left|\nabla v_{\varepsilon m}^{\prime}(t)\right|^{2}+\int_{0}^{t}\left|v_{\varepsilon m}^{\prime \prime}(s)\right|^{2} d s \\
& +\int_{0}^{t}\left|v_{\varepsilon m}^{\prime \prime}(s)\right|_{\Gamma_{0}}^{2} d s+\int_{0}^{t} \alpha(t)\left(\left|v_{\varepsilon m}^{\prime}(s)+\phi^{\prime}(s)\right|^{\rho}, v_{\varepsilon m}^{\prime \prime 2}(s)\right)_{\Gamma_{0}} d s \leq L_{2}
\end{aligned}
$$

where $L_{2}>0$ is independent of $\varepsilon$ and $m$.

\section{Analysis of the Nonlinear Terms.}

From the above estimates we deduce

$$
\begin{gathered}
\left\{v_{\varepsilon m}\right\} \text { is bounded in } L^{2}\left(0, T ; H^{1 / 2}\left(\Gamma_{0}\right)\right), \\
\left\{v_{\varepsilon m}^{\prime}\right\} \text { is bounded in } L^{2}\left(0, T ; H^{1 / 2}\left(\Gamma_{0}\right)\right), \\
\left\{v_{\varepsilon m}^{\prime \prime}\right\} \text { is bounded in } L^{2}\left(0, T ; L^{2}\left(\Gamma_{0}\right)\right) .
\end{gathered}
$$

From (3.13)-(3.15), observing that the imersion $H^{1 / 2}\left(\Gamma_{0}\right) \hookrightarrow L^{2}\left(\Gamma_{0}\right)$ is continuous and compact, and making use of Aubin-Lions theorem, we can extract a subsequence $\left\{v_{\varepsilon \mu}\right\}$ of $\left\{v_{\varepsilon m}\right\}$ such that

$$
v_{\varepsilon \mu} \rightarrow v_{\varepsilon} \text { and } v_{\varepsilon \mu}^{\prime} \rightarrow v_{\varepsilon}^{\prime} \text { a.e. on } \Sigma_{0, T}=\Gamma_{0} \times(0, T) .
$$

Therefore, from (3.16) it follows that

$$
\left|v_{\varepsilon \mu}\right|^{\gamma} v_{\varepsilon \mu} \rightarrow\left|v_{\varepsilon}\right|^{\gamma} v_{\varepsilon} \text { and }\left|v_{\varepsilon \mu}^{\prime}\right|^{\rho} v_{\varepsilon \mu}^{\prime} \rightarrow\left|v_{\varepsilon}^{\prime}\right|^{\rho} v_{\varepsilon}^{\prime} \text { a.e. on } \Sigma_{0, T} \text {. }
$$

On the other hand, from the first and second estimates we obtain

$$
\begin{aligned}
& \left\{\left|v_{\varepsilon \mu}\right|^{\gamma} v_{\varepsilon \mu}\right\} \text { is bounded in } L^{2}\left(\Sigma_{0, T}\right), \\
& \left\{\left|v_{\varepsilon \mu}^{\prime}\right|^{\rho} v_{\varepsilon \mu}^{\prime}\right\} \text { is bounded in } L^{2}\left(\Sigma_{0, T}\right)
\end{aligned}
$$

Thus, combining (3.17)-(3.19), we deduce from Lions' lemma

$$
\left|v_{\varepsilon \mu}\right|^{\gamma} v_{\varepsilon \mu} \rightarrow\left|v_{\varepsilon}\right|^{\gamma} v_{\varepsilon} \text { weakly in } L^{2}\left(\Sigma_{0, T}\right)
$$




$$
\left|v_{\varepsilon \mu}^{\prime}\right|^{\rho} v_{\varepsilon \mu}^{\prime} \rightarrow\left|v_{\varepsilon}^{\prime}\right|^{\rho} v_{\varepsilon}^{\prime} \text { weakly in } L^{2}\left(\Sigma_{0, T}\right)
$$

The above convergences are sufficient to pass to the limit in the nonlinear terms of (3.2) using standard arguments. From this and taking (2.4) into account we obatin

$$
K_{1} y^{\prime \prime}+K_{2} y^{\prime}-\Delta y=0 \text { in } L_{\text {loc }}^{2}\left(0, \infty ; L^{2}(\Omega)\right) .
$$

Moreover, from the generalized Green's formula we infer

$$
\frac{\partial y}{\partial \nu}+y_{t}+\alpha(t)\left(\left|y_{t}\right|^{\rho} y_{t}-|y|^{\gamma} y\right)=0 \text { in } L_{\mathrm{loc}}^{2}\left(0, \infty ; L^{2}\left(\Gamma_{0}\right)\right)
$$

\section{Uniqueness.}

Let $y_{1}$ and $y_{2}$ be strong solutions to problem (1.1). Defining $z=y_{1}-y_{2}$, we deduce from (3.20) and (3.21)

$$
\begin{aligned}
& \left(K_{1} z^{\prime \prime}(t), w\right)+\left(K_{2} z^{\prime}(t) \cdot w\right)+(\nabla z(t), \nabla w)+\left(z^{\prime}(t), w\right)_{\Gamma_{0}} \\
& \quad+\alpha(t)\left(\left|y_{1}^{\prime}\right|^{\rho} y_{1}^{\prime}-\left|y_{2}^{\prime}\right|^{\rho} y_{2}^{\prime}, w\right)_{\Gamma_{0}} \\
& =\alpha(t)\left(\left|y_{2}\right|^{\gamma} y_{2}-\left|y_{1}\right|^{\gamma} y_{1}, w\right)_{\Gamma_{0}},
\end{aligned}
$$

for all $w \in V$.

Substituing $w=z^{\prime}(t)$ in (3.22), we obtain from (H.4)

$$
\begin{aligned}
& \frac{d}{d t}\left\{\frac{1}{2}\left|\sqrt{K_{1}(t)} z^{\prime}(t)\right|^{2}+\frac{1}{2}|\nabla z(t)|^{2}\right\}+\delta\left|z^{\prime}(t)\right|^{2}+\left|z^{\prime}(t)\right|_{\Gamma_{0}}^{2} \\
& \leq \alpha(t)\left(\left|y_{2}\right|^{\gamma} y_{2}-\left|y_{1}\right|^{\gamma} y_{1}, z^{\prime}(t)\right)_{\Gamma_{0}} \\
& \leq C(\gamma) \int_{\Gamma_{0}}\left(\left|y_{2}\right|^{\gamma}+\left|y_{1}\right|^{\gamma}\right)|z(t)|\left|z^{\prime}(t)\right| d \Gamma .
\end{aligned}
$$

Integrating the last inequality over $(0, t)$, using analogous considerations made in the second estimate (see estimate for $I_{2}$ term) and employing Gronwall's lemma, we obtain $\left|z^{\prime}(t)\right|=|\nabla z(t)|=0$. This concludes the proof of uniqueness for strong solutions.

\section{Existence of Weak Solutions.}

Let us consider

$$
\left\{y^{0}, y^{1}\right\} \in V \times L^{2}(\Omega) .
$$

Since

$$
D(-\Delta)=\left\{u \in V \cap H^{2}(\Omega) ; \frac{\partial u}{\partial \nu}=0 \text { on } \Gamma_{0}\right\}
$$


is dense in $V$ and $H_{0}^{1}(\Omega) \cap H^{2}(\Omega)$ is dense in $L^{2}(\Omega)$, there exist $\left\{y_{\mu}^{0}\right\} \subset$ $D(-\Delta)$ and $\left\{y_{\mu}^{1}\right\} \subset H_{0}^{1}(\Omega) \cap H^{2}(\Omega)$ such that

$$
\begin{gathered}
y_{\mu}^{0} \rightarrow y^{0} \text { strongly in } V, \\
y_{\mu}^{1} \rightarrow y^{1} \text { strongly in } L^{2}(\Omega),
\end{gathered}
$$

and, since $\alpha(0)=0$, the compatibility conditions given in (H.2) are verified, that is, for each $\mu \in \mathbf{N}$, one has

$$
\frac{\partial y_{\mu}^{0}}{\partial \nu}+y_{\mu}^{1}=0 \text { on } \Gamma_{0} .
$$

Then, repeating the same arguments used in the first estimate and in the uniqueness of strong solutions, we deduce that there exist $\left\{y_{\mu}\right\}$ a sequence of strong solutions of problem (1.1) and also $y: Q \rightarrow \mathbf{R}$ such that

$$
y_{\mu} \rightarrow y \text { strongly in } C^{0}([0, T] ; V),
$$

$$
y_{\mu}^{\prime} \rightarrow y^{\prime} \text { strongly in } C^{0}\left([0, T] ; L^{2}(\Omega)\right)
$$

and

$$
\left\{\begin{array}{l}
K_{1} y^{\prime \prime}+K_{2} y^{\prime}-\Delta y=0 \text { in } L^{2}\left(0, T ; V^{\prime}\right) \\
y(0)=y^{0} ; y^{\prime}(0)=y^{1}
\end{array}\right.
$$

From now on we are going to define a weak solution to problem (1.1), a function $y$ which verifies (3.27).

\section{Uniform Decay.}

The derivative of the energy defined in (1.3) is given by

$$
\begin{aligned}
e^{\prime}(t)= & -\left(K_{2}(t)-\frac{1}{2} K_{1}^{\prime}(t), y^{2}(t)\right)-\left|y^{\prime}(t)\right|_{\Gamma_{0}}^{2} \\
& -\alpha(t)\left\|y^{\prime}(t)\right\|_{\rho+2, \Gamma_{0}}^{\rho+2}+\alpha(t)\left(|y(t)|^{\gamma} y(t), y^{\prime}(t)\right)_{\Gamma_{0}} .
\end{aligned}
$$

Defining the modified energy by

$$
E(t)=e(t)+\frac{1}{\gamma+2} \alpha(t)\|y(t)\|_{\gamma+2, \Gamma_{0}}^{\gamma+2}
$$


462 M.M. Cavalcanti, V.N.D. Cavalcanti, J.S.P. Filho \& J.A. Soriano

we obtain from the assumptions (H.4), (H.6), (4.1) and from (4.2)

$$
\begin{aligned}
E^{\prime}(t) \leq & -\delta\left|y^{\prime}(t)\right|^{2}-\left|y^{\prime}(t)\right|_{\Gamma_{0}}^{2}-\alpha(t)\left\|y^{\prime}(t)\right\|_{\rho+2, \Gamma_{0}}^{\rho+2} \\
& -\frac{1}{\gamma+2} m_{1} \alpha(t)\|y(t)\|_{\gamma+2, \Gamma_{0}}^{\gamma+2}+2 \alpha(t)\left(|y(t)|^{\gamma} y(t), y^{\prime}(t)\right)_{\Gamma_{0}}
\end{aligned}
$$

Considering the Young's inequality $a b \leq \eta a^{p}+C(\eta) b^{q}$ with $p=\gamma+2$, $q=\frac{\gamma+2}{\gamma+1}$ and $C(\eta)=\eta^{-\frac{1}{\gamma+1}}$ and supposing that $\gamma=\rho$, we deduce

$$
\begin{aligned}
E^{\prime}(t) \leq & -\delta\left|y^{\prime}(t)\right|^{2}-\left|y^{\prime}(t)\right|_{\Gamma_{0}}^{2}-\alpha(t)(1-2 \eta)\left\|y^{\prime}(t)\right\|_{\gamma+2, \Gamma_{0}}^{\gamma+2} \\
& -\alpha(t)\left(\frac{\gamma+1}{\gamma+2}-\eta^{-\frac{1}{\gamma+1}}\right)\|y(t)\|_{\gamma+2, \Gamma_{0}}^{\gamma+2}
\end{aligned}
$$

Choosing $\eta=4^{-(\gamma+1)}$, we have $2\left[4^{-(\gamma+1)}\right]<\frac{1}{2}$ and consequently from (4.4) it follows

$$
\begin{aligned}
E^{\prime}(t) \leq & -\delta\left|y^{\prime}(t)\right|^{2}-\left|y^{\prime}(t)\right|_{\Gamma_{0}}^{2} \\
& -\frac{1}{2} \alpha(t)\left\|y^{\prime}(t)\right\|_{\gamma+2, \Gamma_{0}}^{\gamma+2}-\beta \alpha(t)\|y(t)\|_{\gamma+2, \Gamma_{0}}^{\gamma+2} .
\end{aligned}
$$

where

$$
\beta=\frac{m_{1}}{\gamma+2}-8>0
$$

For every $\varepsilon>0$ we define the perturbed modified energy

$$
E_{\varepsilon}(t)=E(t)+\varepsilon \psi(t)
$$

where

$$
\psi(t)=\int_{\Omega} K_{1} y^{\prime} y d x .
$$

In what follows let $\lambda>0$ be a positive constant such that

$$
|v|^{2} \leq \lambda|\nabla v| ; \forall v \in V
$$

Proposition 4.1. There exists $C_{1}>0$ such that

$$
\left|E_{\varepsilon}(t)-E(t)\right| \leq \varepsilon C_{1} E(t), \quad \forall t \geq 0 \quad \text { and } \quad \forall \varepsilon>0 .
$$


Proof. From (4.7), (4.8) and using Schwarz inequality we infer

$$
|\psi(t)| \leq\left\|K_{1}\right\|_{\infty}^{1 / 2} \lambda^{1 / 2} e(t) \leq\left\|K_{1}\right\|_{\infty}^{1 / 2} \lambda^{1 / 2} E(t)
$$

and from (4.6) we conclude the desired inequality with $C_{1}=\left\|K_{1}\right\|_{\infty}^{1 / 2} \lambda^{1 / 2}$.

Proposition 4.2. There exist $C_{2}>0$ and $\varepsilon_{1}>0$ such that

$$
E_{\varepsilon}^{\prime}(t) \leq-\varepsilon C_{2} E(t) ; \quad \forall t \geq 0 \quad \text { and } \quad \varepsilon \in\left(0, \varepsilon_{1}\right]
$$

Proof. Differentiating $\psi(t)$ with respect to $t$ and replacing $K_{1} y^{\prime \prime}$ by $-K_{2} y^{\prime}+$ $\Delta y$ in the obtained result, it follows that

$$
\psi^{\prime}(t)=\int_{\Omega} K_{1}^{\prime} y^{\prime} y d x-\int_{\Omega} K_{2} y^{\prime} y d x+\int_{\Omega} \Delta y y d x+\int_{\Omega} K_{1}\left|y^{\prime}\right|^{2} d x .
$$

Now, using the generalized Green formula and taking into account that

$$
\frac{\partial y}{\partial \nu}=-y^{\prime}-\alpha(t)\left|y^{\prime}\right|^{\rho} y^{\prime}+\alpha(t)|y|^{\gamma} y
$$

we deduce from (4.9)

$$
\begin{aligned}
\psi^{\prime}(t)= & \int_{\Omega} K_{1}^{\prime} y^{\prime} y d x-\int_{\Omega} K_{2} y^{\prime} y d x-\int_{\Omega}|\nabla y|^{2} d x+\int_{\Omega} K_{1}\left|y^{\prime}\right|^{2} d x \\
& -\int_{\Gamma_{0}} y^{\prime} y d \Gamma-\alpha(t) \int_{\Gamma_{0}}\left|y^{\prime}\right|^{\gamma} y^{\prime} y d \Gamma+\alpha(t) \int_{\Gamma_{0}}|y|^{\gamma+2} d \Gamma .
\end{aligned}
$$

Adding and subtracting the terms $\int_{\Gamma_{0}} K_{1}\left|y^{\prime}\right|^{2} d \Gamma$ and $\alpha(t) \int_{\Gamma_{0}}|y|^{\gamma+2} d \Gamma$ from (4.10), we obtain the following inequality

$$
\begin{aligned}
\psi^{\prime}(t) \leq & -E(t)+2 \int_{\Omega} K_{1}\left|y^{\prime}\right|^{2} d x+2 \alpha(t) \int_{\Gamma_{0}}|y|^{\gamma+2} d \Gamma \\
& +\int_{\Omega} K_{1}^{\prime} y^{\prime} y d x-\int_{\Omega} K_{2} y^{\prime} y d x-\int_{\Gamma_{0}} y^{\prime} y d \Gamma-\alpha(t) \int_{\Gamma_{0}}\left|y^{\prime}\right|^{\gamma} y^{\prime} y d \Gamma .
\end{aligned}
$$

Making use of the inequalities $a b \leq \frac{1}{4 \eta} a^{2}+\eta b^{2}$ and $a b \leq \theta(\eta) a^{p}+\eta b^{q}$, where $\frac{1}{p}+\frac{1}{q}=1$ and $\eta>0$ is arbitrary, and considering (4.8), we conclude from $(4.11)$

$$
\psi^{\prime}(t) \leq-[1-(8+\gamma) \eta] E(t)+2 \alpha(t)\|y(t)\|_{\gamma+2, \Gamma_{0}}^{\gamma+2}
$$


464 M.M. Cavalcanti, V.N.D. Cavalcanti, J.S.P. Filho \& J.A. Soriano

$$
+M_{1}(\eta)\left|y^{\prime}(t)\right|^{2}+M_{2}(\eta)\left|y^{\prime}(t)\right|_{\Gamma_{0}}^{2}+M_{3}(\eta) \alpha(t) \| y^{\prime}(t)||_{\gamma+2, \Gamma_{0}}^{\gamma+2}
$$

where

$$
\begin{aligned}
& M_{1}(\eta)=2\left\|K_{1}\right\|_{\infty}+\frac{\left\|K_{1}^{\prime}\right\|_{\infty}^{2} \lambda^{2}}{4 \eta}+\frac{\left\|K_{2}\right\|_{\infty}^{2} \lambda^{2}}{4 \eta}, \\
& M_{2}(\eta)=\frac{C_{0}^{2}}{4 \eta}\left|y^{\prime}(t)\right| \quad \text { and } \quad M_{3}(\eta)=\theta_{1}(\eta)
\end{aligned}
$$

and $C_{0}>0$ is such that $|y|_{\Gamma_{0}} \leq C_{0}|\nabla y|$.

Choosing $\eta>0$ so that $C_{2}=1-(8+\gamma) \eta>0$ from (4.12) we have

$$
\begin{aligned}
\psi^{\prime}(t) \leq & -C_{2} E(t)+2 \alpha(t)\|y(t)\|_{\gamma+2, \Gamma_{0}}^{\gamma+2} \\
& +M_{1}\left|y^{\prime}(t)\right|^{2}+M_{2}\left|y^{\prime}(t)\right|_{\Gamma_{0}}^{2}+M_{3} \alpha(t)\left\|y^{\prime}(t)\right\|_{\gamma+2, \Gamma_{0}}^{\gamma+2}
\end{aligned}
$$

Taking the derivative in (4.6) with respect to $t$, combining (4.5) and (4.13), it follows that

$$
\begin{aligned}
E_{\varepsilon}^{\prime}(t) \leq & -\left(\delta-\varepsilon M_{1}\right)\left|y^{\prime}(t)\right|^{2}-\left(1-\varepsilon M_{2}\right)\left|y^{\prime}(t)\right|_{\Gamma_{0}}^{2} \\
& -\alpha(t)\left(\frac{1}{2}-\varepsilon M_{3}\right)\left\|y^{\prime}(t)\right\|_{\gamma+2, \Gamma_{0}}^{\gamma+2} \\
& -(\beta-2 \varepsilon) \alpha(t)\|y(t)\|_{\gamma+2, \Gamma_{0}}^{\gamma+2}-\varepsilon C_{2} E(t) .
\end{aligned}
$$

Defining

$$
\varepsilon_{1}=\min \left\{\frac{\delta}{M_{1}}, \frac{1}{M_{2}}, \frac{1}{2 M_{3}}\right\},
$$

then, for all $\varepsilon \in\left(0, \varepsilon_{1}\right]$, we obtain from (4.14) the desired result and, consequently, the Proposition 4.2 is proved.

\section{Proof of the Uniform Decay.}

Let

$$
\varepsilon_{0}=\min \left\{1 / 2 C_{1}, \varepsilon_{1}\right\},
$$

where $C_{1}>0$ is given in Proposition 1 , and let us consider $\varepsilon \in\left(0, \varepsilon_{0}\right]$. As we have $\varepsilon<1 / 2 C_{1}$, we conclude from Proposition 4.1

$$
\frac{1}{2} E(t) \leq E_{\varepsilon}(t) \leq \frac{3}{2} E(t) \leq 2 E(t) ; \quad \forall t \geq 0 .
$$

Consequently $-\varepsilon C_{2} E(t) \leq-\frac{\varepsilon}{2} C_{2} E_{\varepsilon}(t)$ and it follows from Proposition 2

$$
E_{\varepsilon}^{\prime}(t) \leq-\frac{\varepsilon}{2} C_{2} E_{\varepsilon}(t)
$$


Therefore

$$
\frac{d}{d t}\left(E_{\varepsilon}(t) \exp \left(\frac{\varepsilon}{2} t\right)\right) \leq 0
$$

which implies in view of (4.15) that

$$
E(t) \leq 3 E(0) \exp \left(-\frac{\varepsilon}{2} t\right) .
$$

This concludes the proof of Theorem 2.1.

\section{References.}

[1] M.M. Cavalcanti, V.N. Domingos Cavalcanti, J.S. Prates Filho, and J. A. Soriano, Existence and uniform decay of solutions of a degenerate equation with nonlinear boundary damping and boundary memory source term, Nonlinear Analysis T.M.A., 38 (1999), 281-294.

[2] M.M. Cavalcanti, J.S. Prates Filho, and J.A. Soriano, Existence and asymptotic behaviour of a degenerate integro-differential equation with damping, Differential Equations and Dynamical Systems, 7(1) (1999), 67-82.

[3] R. Cipolatti, E. Machtyngier, and E. San Pedro Siqueira, Nonlinear boundary feedback stabilization for Schrodinger equations, Differential and Integral Equations, 9(1) (1996), 137-148.

[4] A. Favini, M.A. Horn, I. Lasiecka, and D. Tataru, Global existence and regularity of solutions to a Von Kármán System with nonlinear boundary dissipation,Differential and Integral Equations, 9(2) (1996), 267-269.

[5] V. Georgiev and G. Todorova, Existence of a solution of the wave equation with nonlinear damping and source terms, Journal of Differential Equations, 109 (1994), 295-308.

[6] A. Haraux and E. Zuazua, Decay estimates for some semilinear damped hyperbolic problem, Arch. Rational Mech. Anal., 100 (1988), 191-206.

[7] J.E. Lagnese and G. Leugering, Uniform stabilization of nonlinear beam by nonlinear boudary feedback, J. Differential Equations, 91 (1991), 355-388. 
466 M.M. Cavalcanti, V.N.D. Cavalcanti, J.S.P. Filho \& J.A. Soriano

[8] N.A. Lar'kin, V.A. Novikov, and N.N. Yanenko, Towards a theory of variable-type nonlinear equations, in 'Numerical Methods in Fluid Dynamics (Yanenko and Shokin, editors),' MIR Publishers, Moscow, (1984), 315-335.

[9] I. Lasiecka, Stabilization of hyperbolic and parabolic systems with nonlinearly perturbed boundary conditions, J. Differential Eqs, 75 (1988), $53-87$.

[10] I. Lasiecka and D. Tataru, Uniform boundary stabilization of semilinear wave equations with nonlinear boundary damping, Differential and Integral Equations, 6(3) (1993), 507-533.

[11] J.L. Lions, Quelques méthodes de résolution des problèmes aux limites non linéaires, Dunod-Gauthier Villars, Paris, 1969.

[12] B. Rao, Stabilization of Kirchhoff plate equation in star-shaped domain by nonlinear boundary feedback, Nonlinear Analysis T.M.A., 20 (1993), 605-626.

[13] E. Zuazua, Uniform stabilization of the wave equation by nonlinear boundary feedback, SIAM J. Control and Optimization, 28 (1990), 466478.

Departamento de Matemática

UNIVERSIDADE ESTADUAL DE MARINGÁ

87020-900 MARINGÁ - PR, BRASIL

RECEIVED MARCH 23, 2000. 